\title{
Crystalline garnet Bragg reflectors for high power, high temperature and integrated applications fabricated by multi-beam Pulsed Laser Deposition
}

Katherine A. Sloyan, Timothy C. May-Smith, Michalis N. Zervas, Robert W. Eason Optoelectronics Research Centre, University of Southampton, Southampton UK SO17 $1 B J$

kas@orc.soton.ac.uk

\begin{abstract}
Crystalline Bragg reflectors are of interest for high power, high temperature and integrated applications. We demonstrate the automated growth of such structures by shuttered multi-beam Pulsed Laser Deposition (PLD). Geometries include 145 layer stacks exhibiting $>99.5 \%$ reflection and $\pi$ phase-shifted designs. A crystalline grating strength-apodized sample was grown by mixing plumes to obtain layers with custom refractive indices. Peak reflection wavelength was tuneable with incident position, samples withstood temperatures of $\sim 750^{\circ} \mathrm{C}$ and film and substrate have been shown to withstand incident pulsed laser fluences of up to $\sim 33 \mathrm{Jcm}^{-2}$.
\end{abstract}

Bragg gratings are useful in a range of applications, from mirrors and filters to sensors. They are usually fabricated from amorphous materials, and hence their use can be limited in high power or high temperature applications. Epitaxial crystal Bragg structures however, with their generally higher melting points and thermal conductivities, should be much more resistant to such damage. Such structures are also of interest for integrated growth of hybrid laser crystals: epitaxial mirrors may be grown directly onto a thin-disc laser, for example. In this paper we present a method for the simple growth of such structures, suitable for rapid prototyping: shuttered multi-beam, multi-target Pulsed Laser Deposition (PLD). 
Single-beam, single-target PLD has been used previously to demonstrate growth of both crystalline and amorphous Bragg multilayers [1-4]. In general however, little emphasis has been placed on speed or simplicity of fabrication, or on mirror or filter applications. As we demonstrate in this paper, shuttered multi-beam PLD extends the basic PLD setup to allow automated growth of epitaxial Bragg stacks in both simple quarter-wave (with over 100 layers) and sophisticated apodized geometries, the latter of which in particular are very difficult to achieve by other methods.

Depositions were carried out using the shuttered multi-beam PLD setup previously demonstrated for superlattice growth [5]. Bursts of pulses ablated each target in turn or simultaneously, with the number of pulses per burst determined by the shutter open/close timings. Samples were composed of $\mathrm{Y}_{3} \mathrm{Al}_{5} \mathrm{O}_{12}(\mathrm{YAG})$ and $\mathrm{Gd}_{3} \mathrm{Ga}_{5} \mathrm{O}_{12}(\mathrm{GGG})$ layers grown on single-crystal YAG substrates of size $10 \times 10 \times 1 \mathrm{~mm}^{3}$. Substrates were heated via $\mathrm{CO}_{2}$ laser to temperatures of $\sim 750^{\circ} \mathrm{C}$ to obtain epitaxially-orientated crystal films. Deposition took place in an $\mathrm{O}_{2}$ gas pressure of $1 \times 10^{-2}$ mbar and target-substrate distance was $\sim 40 \mathrm{~mm}$. Excimer (wavelength $248 \mathrm{~nm}$ ) and frequency-quadrupled Nd:YAG (wavelength $266 \mathrm{~nm}$ ) lasers of fluence $\sim 2.5 \mathrm{Jcm}^{-2}$ and $10 \mathrm{~Hz}$ repetition rate ablated single-crystal YAG and GGG targets respectively. Targets were tilted over a range of $8^{\circ}$ during deposition and the substrate was rotated to aid homogenous film growth. A compromised common set of deposition conditions was chosen for ease of fabrication and to allow mixed layer growth for gratingstrength apodisation. Layers consequently exhibited stoichiometry and hence refractive index $n$ that did not match bulk values, due to gallium and aluminium loss in the cases of GGG and YAG respectively. Refractive index values were inferred by fitting of transmission spectra as 
$\sim 1.9$ and $\sim 2.0$ for YAG and GGG layers respectively, compared with bulk values of $n_{Y A G}=1.82[6]$ and $n_{G G G}=1.95[7]$.

A number of designs were implemented. Quarter-wave stacks consisted of alternating layers of thickness $\lambda / 4 n$, while $\pi$ phase-shifted structures consisted of quarter-wave stacks either side of a spacer layer of thickness $\lambda / 2 n$. These structures were grown automatically, with shutter open/close timing controlled via custom LabVIEW programs. Grating strength apodisation [8] was also achieved by mixing plumes to obtain layers with custom refractive indices. This sample consisted of alternating layers of YAG and mixed YAG/GGG, varied in ratios of 9:1, 8:2 and so on to $0: 10$ and back to $9: 1$ by changing the relative repetition rates of the ablating lasers using the shutters, to obtain an approximately Gaussian grating strength profile. The optical thickness was approximately the same for each layer, resulting in a structure that was grating-strength apodized only (not chirped-apodized). As such, the physical thickness of each mixed layer was varied to compensate for the change in index.

With no in-situ diagnostics to measure thickness during deposition, deposition rates were inferred via spectrometry of single-material test films (a value for optical thickness can be obtained by comparing positions of interference peaks [9]). Models of quarter-wave and $\pi$ phase-shifted geometries were created in MATLAB. These models incorporated chirp (i.e. a linear variation in thickness) and loss (absorption or scattering) but not fluctuations in thickness.

The majority of spectra presented were obtained with a Varian Cary 500 white light spectrophotometer operating in transmission mode with a circular aperture of $\sim 1 \mathrm{~mm}$. Results were found to match those obtained using a CRAIC 20/20 PV microscope-coupled 
spectrometer, used in reflection mode. Absorption and scattering were hence unlikely to be significant, and it was assumed that $\mathrm{R}=1-\mathrm{T}$, where $\mathrm{R}$ and $\mathrm{T}$ are reflection and transmission intensity coefficients respectively. In the case of $\pi$ phase-shifted structures, where it was suspected that the short coherence length of white light was limiting feature resolution, a transmission spectrum was also obtained using a tuneable Ti:sapphire laser. X-ray diffraction was undertaken with a Siemens D5000 powder diffractometer.

The transmission spectrum of an example of a quarter-wave Bragg stack with 67 layers and a design peak wavelength of $946 \mathrm{~nm}$ is shown in figure 1 along with an approximate fit for comparison. The model assumes no absorption or scattering loss and a slight chirp (a decrease in thickness of $\sim 1 \%$ over the course of deposition). The measured peak is not completely symmetrical and hence cannot be fitted perfectly, and peak definition is also poorer than expected, suggesting that there may be significant factors that were not included in the model. These may include different chirp rates of the two materials, due to different laser energy drift or target degradation rates, and fluctuations in layer thickness due to corresponding fluctuations in pressure or laser energy during the deposition.

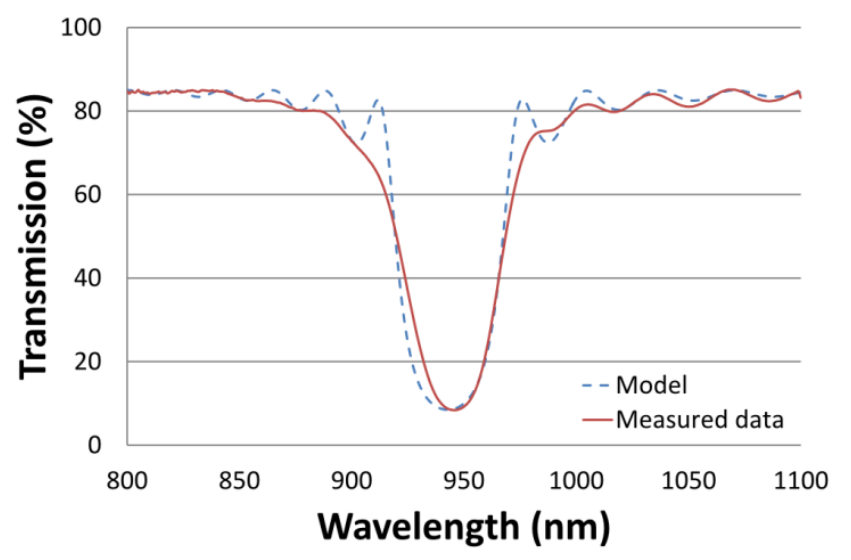

Figure 1 Measured spectrum of a 67 layer quarter-wave stack with fit from MATLAB model for comparison 
Figure 2 shows the transmission spectrum of a quarter-wave stack with 145 alternating layers, also with a design peak wavelength of $946 \mathrm{~nm}$. Transmission is $<0.5 \%$, corresponding to a peak reflection of $>99.5 \%$. An approximate fit is shown for comparison, which implies that layer thicknesses decreases linearly by around $2 \%$ over the course of the $\sim 6$ hour deposition. Such a drift would be relatively easy to correct in the case of the excimer laser; however, the difficulty in quickly retuning the harmonic crystals in the case of the Nd:YAG lasers renders this more problematic. Sub-peaks such as those shown by the model cannot be observed easily in the measured data, and where small peaks can be seen (i.e. 1000-1100 nm region) the peak positions do not match those of the model. This latter may be the result of different chirping for each material or fluctuations in the growth rates.

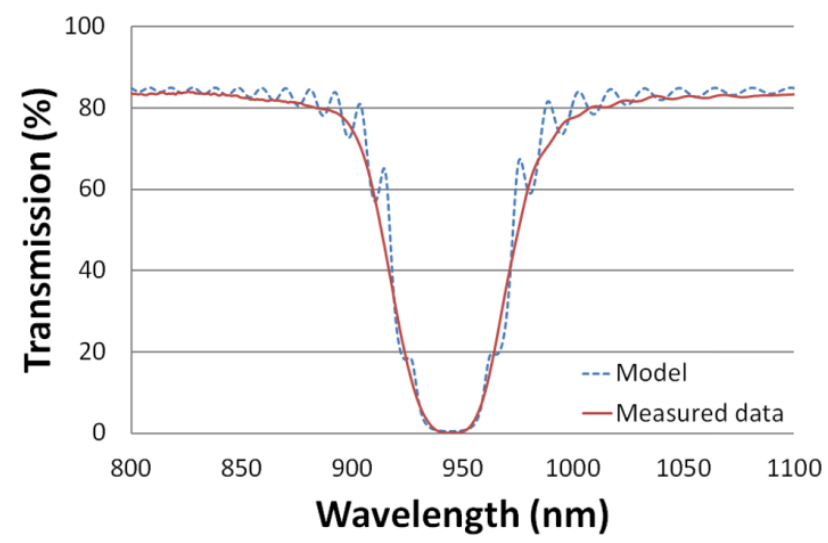

Figure 2 Measured spectrum of a 145 layer quarter-wave stack with fit from MATLAB model for comparison

Peak wavelength can be tuned over $\sim 60 \mathrm{~nm}$ due to a predictable change in film thickness across the sample surface (a result of film curvature). While control of this curvature is limited in the case of the YAG/GGG layers grown in this experiment, the use of materials with a wider window of overlapping conditions would allow much greater control. As expected, only peaks corresponding to the (400) and (800) peaks of YAG and GGG can be observed in X-ray diffraction spectra, confirming that crystal growth was epitaxial on the 
YAG substrate, the XRD spectrum of which also showed only (400) and (800) peaks. The XRD spectrum of an example stack (145 layers) is displayed in figure 3.

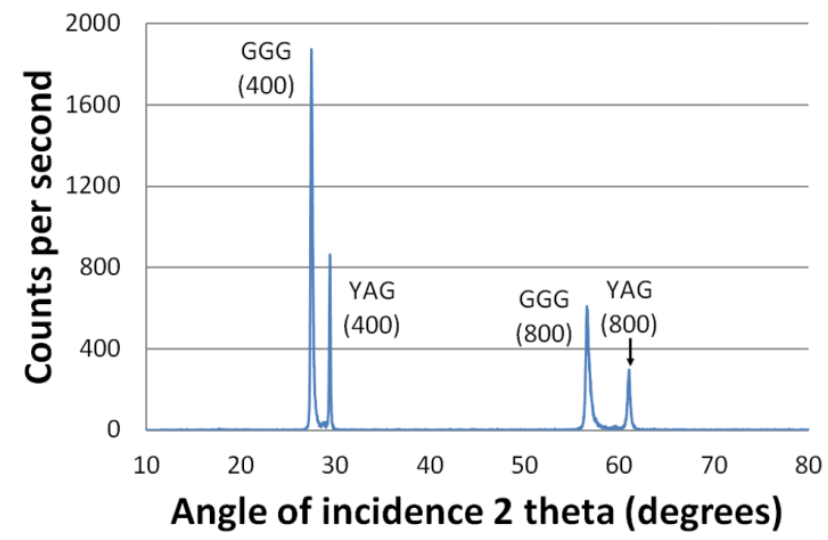

Figure 3 X-ray diffraction spectrum of an example stack (145 layers). Only (400) and (800) peaks can be observed, indicating that the film was epitaxially orientated.

$\pi$ phase-shifted stacks consisted of two 21 layer stacks of alternating YAG and GGG either side of a YAG layer of thickness $\lambda / 2 n$. An ideal structure acts as a Fabry-Perot cavity, resulting in a spectrally narrow reflection trough in the centre of an otherwise standard quarter-wave reflection peak [8]. The measured transmission spectrum is shown in figure 4. An increase of transmission of $\sim 50 \%$ can be observed in the centre of the main transmission trough. Comparison with the model suggests that loss and chirp are not significant for this sample; however, the fact that the transmission value did not increase to that of the substrate only $(\sim 83 \%)$ implies that the structure is not a perfect cavity, and there were undesired fluctuations in the optical thicknesses of the stacks either side. The spectrum described in figure 4 was obtained using a tuneable Ti:sapphire laser. 


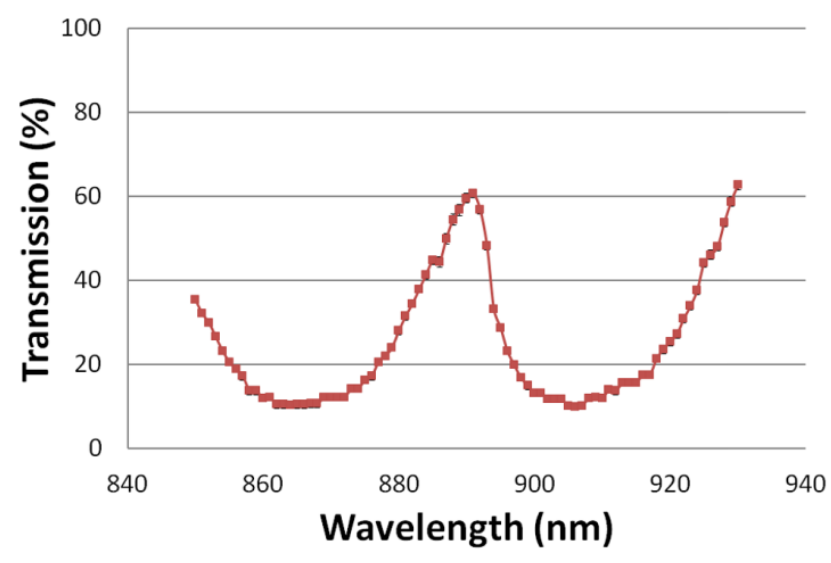

Figure 4: Transmission spectrum of a $\pi$ phase-shifted stack showing the characteristic increase in transmission at $\sim 890 \mathrm{~nm}$, in the centre of the main peak.

The spectra of the apodized sample, and a quarter-wave Bragg stack of similar peak height deposited under the same conditions, can be seen in figure 5. As expected, side bands are suppressed relative to the quarter-wave stack case without significant increase in central peak width. As previously, only XRD peaks corresponding to the (400) and (800) orientations of the YAG, GGG and mixed layers were observed, following the orientation of the substrate; the sample was hence epitaxially-orientated crystal. Peaks representing diffraction from each of the eleven materials in the sample (YAG, GGG and mixtures) can be observed in figure 6, as can a peak corresponding to the underlying substrate.

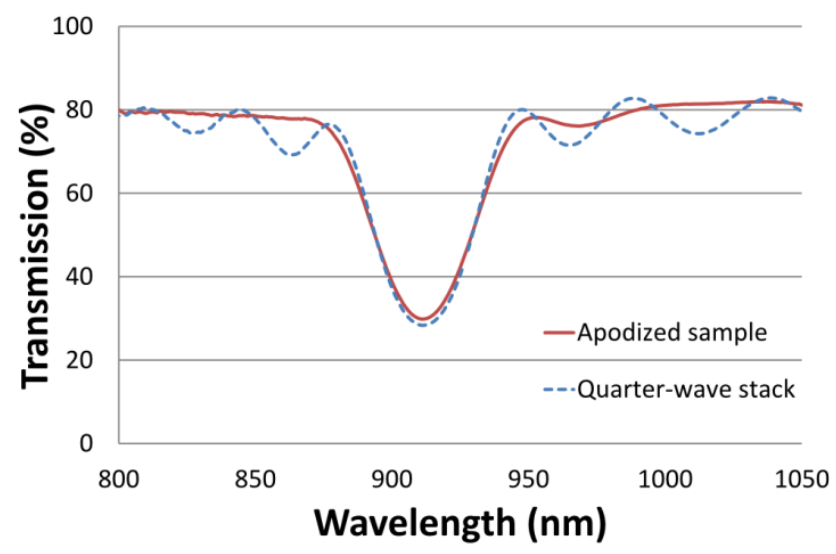

Figure 5 Transmission spectra of apodized and quarter-wave stacks. Side bands are clearly suppressed in the apodized case. 


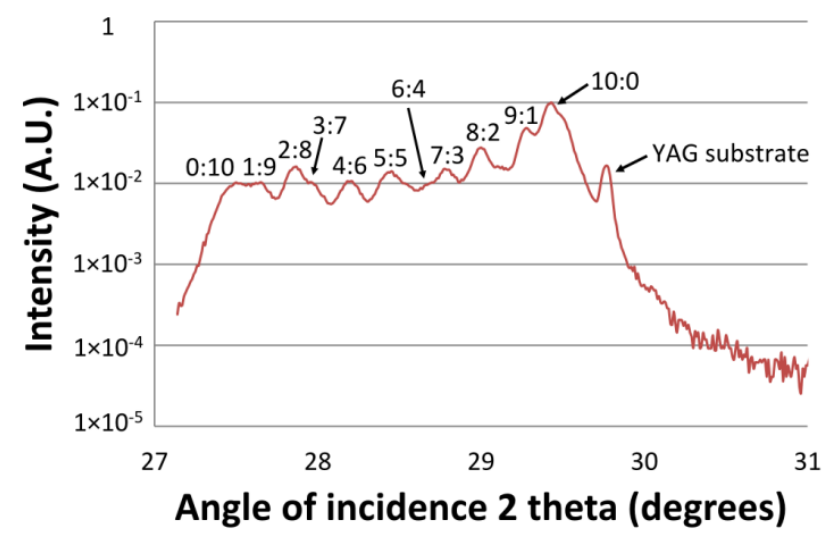

Figure 6 Partial X-ray diffraction spectrum of an apodized sample showing (400) contributions from each of the mixed layers (ratios labelled) as well as the underlying YAG substrate.

A 45-layer quarter-wave stack was subjected to preliminary pulsed laser damage testing using a $70 \mathrm{kHz}$ SPI redENERGY fibre laser operating at $\sim 1060 \mathrm{~nm}$. Bursts of $\sim 75$ pulses irradiated a line on the sample surface and any subsequent damage was recorded. The probability of damage occurring was non-zero above a fluence of $\sim 33 \mathrm{Jcm}^{-2}$ for both the film and bulk substrate. This is already on a par with many of the best commercially available high-energy mirrors, and the threshold is expected to increase by at least a factor of two with the use of improved substrates (reported damage thresholds of bulk YAG range from $100 \mathrm{Jcm}^{-2}$ [10] to an order of magnitude higher [11]).

Samples are known to withstand heating to $750{ }^{\circ} \mathrm{C}$, the growth temperature. A 45-layer quarter-wave grating was subjected to $\mathrm{CO}_{2}$ laser annealing at $\sim 1000{ }^{\circ} \mathrm{C}$ for $\sim 1$ hour, with ramping periods of $\sim 30$ minutes. Annealing took place in an argon atmosphere at a pressure of $1 \times 10^{-2}$ mbar. Transmission spectra before and after annealing are shown in figure 7 . The difference in the pre- and post-annealing spectra suggests that some diffusion between layers has taken place, as might be expected for garnet layers at such temperatures [12]. This investigation should be extended to intermediate annealing temperatures with all three sample geometries to determine at what temperature interdiffusion begins to limit device 
performance, and whether this critical temperature is the same for $\pi$ phase-shifted and apodised stacks. A different choice of materials, sesquioxides for example, may also be investigated, as a grating composed of such material may be capable of withstanding temperatures at which significant interdiffusion of garnet layers occurs.

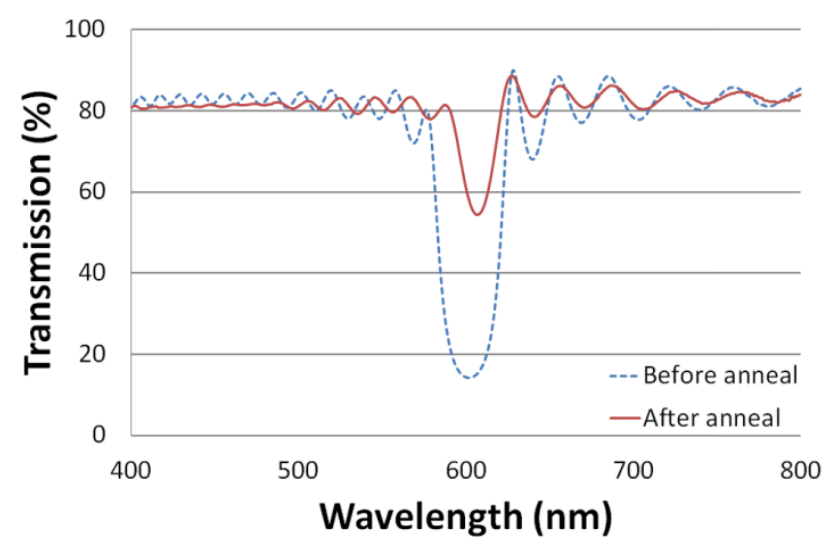

Figure $7 \mathrm{Spectra}$ of a quarter-wave stack before and after annealing in argon for one hour at $\sim 1000^{\circ} \mathrm{C}$.

As mentioned above, sample performance was likely limited by layer-to-layer thickness variation. These could be caused by a number of factors, including fluctuations in laser energy or ambient gas pressure, which influence both growth rate and stoichiometry. The introduction of in-situ diagnostics would help mitigate this problem: a low power laser could be used to perform reflectivity measurements during deposition [13] which could be combined with the LabVIEW control programs to automatically stop deposition when a layer has reached the desired thickness. It is important to remember when introducing additional diagnostics, however, that experimental flexibility or growth rate and/or turnaround time should ideally not be sacrificed: these are the real advantages of the shuttered PLD technique.

To conclude, crystalline Bragg reflectors are of interest for high power, high temperature and integrated applications, due to the high damage thresholds and the potential for integrated epitaxial growth. Such structures have been fabricated quickly and easily in various 
geometries via shuttered multi-beam PLD. Designs included 145 layer stacks exhibiting $>99.5 \%$ reflection as well as $\pi$ phase-shifted geometries. A crystalline apodized sample with an approximately Gaussian grating-strength profile was grown by mixing plumes to obtain a custom refractive index for each layer. Due to a predictable variation in layer thickness across the sample surface, peak reflection wavelength was tuneable via changing incident position. YAG and GGG were used as they had the largest index contrast of the garnets available, but the compromised conditions required for automated growth resulted in layers with stoichiometry far from bulk. While samples were grown at $\sim 750{ }^{\circ} \mathrm{C}$, this choice of materials and conditions may have diminished the ability of the structures to withstand temperatures $\sim 1000{ }^{\circ} \mathrm{C}$. High power laser damage testing, however, suggests that the damage threshold of the crystal mirrors should be very similar to that of bulk crystal. Samples have already been shown to withstand fluences of up to $\sim 33 \mathrm{Jcm}^{-2}$, and with the use of higher quality crystal substrates, damage thresholds expected to increase further.

Acknowledgements: This work was funded by the Engineering and Physical Sciences Research Council (EPSRC) under grant nos. EP/J008052/1 and EP/F019300/1. The authors are grateful for the help of Amol Choudhary (ORC) in obtaining Ti:sapphire spectra and to SPI Lasers for access to pulsed fibre laser testing facilities. The authors would also like to thank Prof. Mark T. Weller and Dr Mark E. Light for access to X-ray diffraction facilities in the School of Chemistry at the University of Southampton. K. A. Sloyan would like to acknowledge the support of an EPSRC studentship.

\section{References:}

[1] S. Weissmantel, G. Reisse, B. Keiper, U. Broulik, H. Johansen and R. Scholz, Appl. Surf. Sci 108(1) 1 (1997) 
[2] J. Sellmann, Ch. Sturm, R. Schmidt-Grund, Ch. Czekalla, J. Lenzner, H. Hochmuth, B. Rheinländer, M. Lorenz and M. Grundmann, Phys. Status Solidi (C) 5(5) 1240 (2008)

[3] S. Kahl, and A.M. Grishin, Phys. Rev. B 71205110 (2005)

[4] S. I. Khartsev and A. M. Grishin Appl. Phys. Lett. 87122504 (2005)

[5] K.A. Sloyan, T.C. May-Smith, M. Zervas, R.W. Eason, S. Huband, D. Walker and P.A. Thomas, Opt. Ex. 18(24) 24679 (2010)

[6] R. Adair, L. L. Chase and S. A. Payne, Phys. Rev. B 39(5) 3337 (1989)

[7] D. L. Wood, and K. Nassau, Appl. Opt. 29(25) 3704 (1990)

[8] W.H. Loh, M.J. Cole, M.N. Zervas, S. Barcelos, and R.I. Laming, Opt. Lett. 20(20) 2051 (1995)

[9] R. Swanepoel, J. Phys. E: Sci. Instrum. 161214 (1983)

[10] J. Bisson, Y. Feng, A. Shirakawa, H. Yoneda, J. Lu, H. Yagi, T. Yanagitani, and K. Ueda, Jpn. J. Appl. Phys. 421025 (2003)

[11] B.T. Do and A.V. Smith, Appl. Opt. 48(18) 3509 (2009)

[12] M. Y. Chern, C. C. Fang, J. S. Liaw, J. G. Lin, C. Y. Huang, Appl. Phys. Lett. 69(6) 854 (1996)

[13] R. Serna and C. N. Afonso Appl. Phys. Lett. 69(11) 1541 (1996) 